\title{
Assessment of macular choroidal thickness by optical coherence tomography and angiographic changes in central serous chorioretinopathy.
}

\section{AUTHOR(S):}

Jirarattanasopa, Pichai; Ooto, Sotaro; Tsujikawa, Akitaka; Yamashiro, Kenji; Hangai, Masanori; Hirata, Masaya; Matsumoto, Akiko; Yoshimura, Nagahisa

\section{CITATION:}

Jirarattanasopa, Pichai ... [et al]. Assessment of macular choroidal thickness by optical coherence tomography and angiographic changes in central serous chorioretinopathy. Ophthalmology 2012, 119(8): 1666-1678

\section{ISSUE DATE:}

2012-08

URL:

http://hdl.handle.net/2433/159946

\section{RIGHT:}

(C) 2012 American Academy of Ophthalmology. Published by Elsevier Inc.; This is not the published version. Please cite only the published version.; この論文は出版社版でありません。引用の際には出版社版を ご確認ご利用ください。 


\section{Assessment of Macular Choroidal Thickness by Optical Coherence Tomography and Angiographic Changes in Central Serous Chorioretinopathy}

Jirarattanasopa Pichai, MD, ${ }^{1,2}$ Sotaro Ooto, $M D,{ }^{2}$ Akitaka Tsujikawa, $M D,{ }^{2}$ Kenji Yamashiro, $M D,{ }^{2}$ Masanori Hangai, $M D,{ }^{2}$ Masaya Hirata, $M D,{ }^{2}$ Akiko Matsumoto, $M S,{ }^{2,3}$ Nagahisa Yoshimura, $M D^{2}$

Running head: Macular choroidal thickness and angiographic changes in CSC

Author affiliations: From the ${ }^{1}$ Department of Ophthalmology, Faculty of Medicine, Prince of Songkla University, Songkla, Thailand; ${ }^{2}$ Department of Ophthalmology and Visual Sciences, Kyoto University Graduate School of Medicine, Kyoto, Japan; ${ }^{3}$ Topcon Corporation, Tokyo, Japan

This article contains material for online-only use. The following should appear online-only: Supplemental Tables 1-3.

Financial Support: This research was partially supported by a Grant-in-Aid for Scientific Research (21796179) from the Japan Society for the Promotion of Science (JSPS) and Topcon Inc. (Tokyo, Japan). 
1

2 3 4

Conflict of Interest: Masanori Hangai and Nagahisa Yoshimura are paid members of the advisory boards of Topcon Inc., Akiko Matsumoto is an employee of Topcon Inc.

Correspondence: Sotaro Ooto, MD, Department of Ophthalmology and Visual Sciences, Kyoto University Graduate School of Medicine, 54 Kawahara-cho, Shogoin, Sakyo-ku, Kyoto 606-8507, Japan

Telephone: +81-75-751-3248; Facsimile: +81-75-752-0933

E-mail: ohoto@kuhp.kyoto-u.ac.jp 


\section{Abstract}

Objective: To investigate the relationship between macular choroidal thickness measured by high-penetrating swept-source optical coherence tomography (SS-OCT) and angiographic findings in central serous chorioretinopathy (CSC).

Design: Prospective cross-sectional case series.

Participants and Controls: Forty-four patients with CSC (34 eyes) and 17 volunteer subjects (17 normal eyes).

Methods: All subjects underwent a comprehensive ophthalmologic and SS-OCT prototype examination. All patients with CSC also underwent simultaneous fluorescein angiography (FA) and indocyanine green angiography (IA). Mean regional choroidal thickness measurements on the Early Treatment Diabetic Retinopathy Study (ETDRS) layout and squared sector grids were obtained by three-dimensional raster scanning using SS-OCT.

Main Outcome Measures: Macular choroidal thickness and angiographic abnormalities.

Results: Mean whole macular choroidal thickness in eyes with CSC (total, $329.3 \pm 83.0$ $\mu \mathrm{m}$; classic CSC, $326.9 \pm 83.1 \mu \mathrm{m}$; chronic CSC, $325.4 \pm 93.3 \mu \mathrm{m}$; and multifocal posterior pigment epitheliopathy, $359.0 \pm 15.5 \mu \mathrm{m}$ ) was greater than that in normal eyes $(233.0 \pm 67.0 \mu \mathrm{m})(P<0.001)$. In unilateral cases, mean whole macular choroidal thickness was greater in eyes with unilateral CSC than in unaffected fellow eyes $(P=$ 0.021). There was no significant difference in choroidal thickness between active eyes and resolved eyes in any of the ETDRS sectors. Mean choroidal thickness was greater in areas with leakage on FA than in areas without leakage $(P=0.001)$. Mean choroidal 
1

2 3 4

thickness was greater in areas with choroidal vascular hyperpermeability and in areas with punctate hyperfluorescent spots on IA than in unaffected areas $(P<0.001$ for both).

Conclusions: Increased choroidal thickness was observed in the whole macular area of eyes with any of the CSC subtypes. Choroidal thickness was related to leakage from the retinal pigment epithelium, choroidal vascular hyperpermeability, and punctate hyperfluorescent lesions. These findings provide evidence that CSC may be caused by increased hydrostatic pressure in the choroid. 
Central serous chorioretinopathy (CSC) is characterized by serous retinal detachment (SRD) in the macula, often accompanied by small serous pigment epithelial detachments and retinal pigment epithelium (RPE) atrophy. Indocyanine green angiography $(\mathrm{IA})$ shows filling delay in choroidal arteries in addition to choriocapillaris, ${ }^{(1-6)}$ vascular dilatation, ${ }^{(2,4,5)}$ multiple areas of choroidal vascular hyperpermeability, ${ }^{(1-17)}$ and punctuate hyperfluorescent spots. ${ }^{(14)}$ These angiographic findings strongly suggest that the principle mechanism underlying CSC involves choroidal vascular changes. ${ }^{(1-14)}$

Imaging of the choroid with commercially available optical coherence tomography (OCT) does not allow visualization of the entire choroidal structure, due to its low penetration and high backscattering at the level of the RPE. However, since Spaide and associates ${ }^{(15)}$ introduced enhanced depth imaging (EDI) OCT, an increasing number of investigators have studied choroidal thickness in healthy and diseased eyes. ${ }^{(16-25)}$ In fact, using the EDI-OCT technique, it has been reported that subfoveal choroidal thickness is increased in patients with CSC compared with patients with normal eyes. ${ }^{(18,19)}$ However, EDI-OCT is usually coupled to multiple averaging to achieve high contrast and low speckle noise, resulting in less detailed raster scan images. For this reason, previous studies have focused on subfoveal choroidal thickness only. On the other hand, the relationship between choroidal thickness and angiographic changes in those eyes remains elusive.

Recently, other investigators reported the measurement of choroidal thickness with the use of OCT at a longer wavelength. ${ }^{(26-28)}$ In these more recent studies, higher penetration of the OCT probe light, which uses a center wavelength of approximately $1000 \mathrm{~nm}$ instead of the current OCT probing light operated at approximately $800 \mathrm{~nm}$, 
allows us to visualize the entire choroid without resorting to the EDI system or multi-averaging. ${ }^{(26,27)}$ Swept-source (SS)-OCT at a longer wavelength, which is characterized by a high-speed scan rate and a relatively low sensitivity roll-off versus depth compared with the spectral-domain OCT, allows us to obtain a three-dimensional (3D) high-contrast image of the choroid. ${ }^{(29-34)}$

In the study described hereafter, we scanned the whole macular area of eyes with CSC by high-penetrating SS-OCT, using a 3-D raster scan protocol, and produced a choroidal thickness map of the macular area. By applying both the sectors used by the Early Treatment Diabetic Retinopathy Study (ETDRS) and square grid sectors to this map, we measured the mean choroidal thickness in each area. In addition, we investigated the relationship between choroidal thickness and angiographic changes.

\section{METHODS}

All investigations adhered to the tenets of the Declaration of Helsinki, and the study was approved by the institutional review board and the ethics committee at Kyoto University Graduate School of Medicine. After the nature of and possible consequences of the study were explained, written informed consent was obtained from all participating subjects.

\section{Subjects}

This prospective cross-sectional study enrolled 34 patients (31 men and 3 women; 44 eyes) with CSC but without any other macular abnormality, who visited the Macular Service at Kyoto University Hospital, Kyoto, Japan, between October 2010 and March 2011, and 17 volunteers ( 9 men and 8 women; 17 eyes) with no eye disease. 
All subjects underwent a comprehensive ophthalmologic examination, including measurement of best-corrected visual acuity (BCVA) and intraocular pressure, autorefractometry/keratometry, indirect ophthalmoscopy, slit-lamp biomicroscopy with a contact lens, color fundus photography, and SS-OCT with a prototype system. All patients with CSC also underwent simultaneous fluorescein angiography (FA) and IA using a confocal laser scanning ophthalmoscope (HRA2; Heidelberg Engineering, Dossenheim, Germany). The diagnosis of CSC was based on results of the fundus examination, FA, IA, and OCT and exclusion of neovascular maculopathy (i.e., age-related macular degeneration, polypoidal choroidal vasculopathy, pathologic myopia, idiopathic choroidal neovascularization, and angioid streaks) and other causes of SRD (i.e., intraocular inflammation, posterior segment tumor, and drusen).

Exclusion criteria included history of intraocular surgery including cataract surgery and photodynamic therapy (PDT), except for retinal photocoagulation; evidence of glaucoma or high intraocular pressure ( $\geq 22 \mathrm{~mm} \mathrm{Hg}$ ); and poor image due to dense cataract or unstable fixation. Subjects with systemic diseases or conditions that might affect choroidal thickness were also excluded, such as those with diabetes mellitus or malignant hypertension, or those who were pregnant.

\section{Classification of CSC}

CSC patients were classified into 3 types: classic CSC, chronic CSC, and multifocal posterior pigment epitheliopathy (MPPE). ${ }^{(35)}$ Classic CSC was defined as one or only few specific points of angiographic leakage from the RPE. ${ }^{(13)}$ Chronic CSC was defined as broad areas of granular hyperfluorescence during FA associated with many indistinct 
areas of leakage. ${ }^{(13)}$ MPPE was defined as multiple massive leakages from the choroid into the subretinal space. ${ }^{(35,36)}$

All types of CSC eyes were divided into active and resolved eyes. Active eye was defined as serous retinal detachment involving the macula. Resolved eye was defined by the absence of serous retinal detachment at the time of measurement.

Choroidal filling delay was observed in the early phase of IA, and choroidal hyperpermeability and punctate hyperflourescent spots were determined in the late phase of IA. All these angiographic features and classifications were evaluated by an experienced ophthalmologist (SO) who was unaware of the SS-OCT results.

\section{SS-OCT system and scan protocols}

We used an SS-OCT prototype system (Topcon, Tokyo, Japan) with an axial scan rate of $100,000 \mathrm{~Hz}$ operated at the $1-\mu \mathrm{m}$ wavelength region. ${ }^{(34)}$ In the current SS-OCT system, the light source was a wavelength-sweeping laser with a tuning range of approximately $100 \mathrm{~nm}$ centered at $1050 \mathrm{~nm}$, yielding $8-\mu \mathrm{m}$ axial resolution in tissue. Transverse resolution was set to approximately $20 \mu \mathrm{m}$. A single OCT image consisting of $1000 \mathrm{~A}$ lines can be acquired in $10 \mathrm{~ms}$. SS-OCT imaging of the cornea was conducted at $1050 \mathrm{~nm}$ with approximately $1 \mathrm{~mW}$ power, which is well below the safe retinal exposure limit established by the American National Standards Institute. Sensitivity was measured to be approximately $98 \mathrm{~dB}$ at this input power.

SS-OCT examinations were performed by trained examiners after pupil dilation. First, horizontal and vertical line scans $(12 \mathrm{~mm})$ through the fovea were obtained, and $\sim 50$ B-scan images were averaged to reduce speckle noise. Second, a 3D imaging data set was acquired for each subject with a raster scan protocol of 512 (horizontal) $\times 128$ 
(vertical) A-scans per data set (total, 65,536 axial scans/volume) in $0.8 \mathrm{~s}$. Each 3D scan covered an area of $6 \times 6 \mathrm{~mm}$ centered on the fovea, which was confirmed by an internal-fixation and fundus camera integrated in the instrument. To reduce speckle noise, each image was denoised by applying the weighted moving average from 3 consecutive images. ${ }^{(34)}$ Owing to the invisible scanning light and high-speed scanning, eye movement during the 3D-scan was minimal.

\section{Choroidal thickness measurement}

The choroidal thickness was measured as the distance between Bruch's membrane (or the outer border of the RPE-Bruch's membrane complex) and the chorioscleral border (Fig. 1). In each image of the 3D data set, both lines were determined manually by a trained ophthalmologist (JP) who was blinded to the diagnosis, classification, or angiographic findings. Automated built-in calibration software was used to determine the distance between the 2 lines. From all 128 images of each 3D data set, a choroidal thickness map of $6 \times 6 \mathrm{~mm}$ was created. After the choroidal thickness map was created, the ETDRS sectors were applied to it (Fig. 1A). First, the central fovea on each image was manually registered, if necessary, to coincide with the central circle on the ETDRS segmentation diagram. The mean thickness of each sector was measured in 4 "inner ring" sectors (superior, inferior, nasal, and temporal) 1-3 $\mathrm{mm}$ from the center of the fovea, and in 4 "outer ring" sectors (superior, inferior, temporal, and nasal) 3-6 mm from the center of the fovea. A $5 \times 5$ grid sector of $5 \times 5 \mathrm{~mm}$ was further applied to the thickness map. The $5 \times 5 \mathrm{~mm}$ grid divided the macular region into 25 square areas (Fig. 1B). This grid sector was used for comparison with the angiographic findings. Sectors with angiographic abnormalities such as choroidal filling delay and choroidal vascular 
hyperpermeability were defined as those that involved angiographic abnormalities in more than $50 \%$ of the area.

\section{Statistical analyses}

A $t$-test was used for comparison of variables associated with mean choroidal thickness between normal eyes and eyes with CSC, eyes with unilateral CSC and unaffected fellow eyes, unaffected fellow eyes with and without choroidal hyperpermeability, active eyes and resolved eyes, and areas with angiographic changes and areas without them. A $t$-test was also used for a comparison of age and refractive error between normal eyes and CSC eyes, and active eyes and resolved eyes. The Tukey-Kramer test was used for comparison of age and refractive error, and for comparison of mean choroidal thickness between CSC subtypes. We calculated the Pearson's correlation coefficient and partial correlation to examine associations between choroidal thickness and age or refractive error. All statistical evaluations were performed using commercially available software programs (SPSS2; SPSS Japan, Tokyo, Japan) (StatMatelll; ATMS, Tokyo, Japan). A $P$ value of less than 0.05 was considered to indicate statistical significance.

\section{RESULTS}

In the present study, 44 eyes of 34 patients and 17 eyes of 17 healthy volunteers were examined. The mean age was $57.3 \pm 13.7$ years in eyes with CSC, and $62.1 \pm 15.4$ years in normal eyes $(P=0.242$, by the $t$-test). The mean refractive error was $-0.8 \pm 2.5$ diopters in eyes with CSC, and $0.1 \pm 2.5$ diopters in normal eyes $(P=0.245$, by the $t$-test). Among patients with CSC, 19 (56\%) had unilateral evidence or history of serous retinal detachment. Twenty-three eyes showed classic CSC, 17 showed chronic CSC, 
and 4 had MPPE. Twenty-seven (61\%) eyes were classified as active, and 17 (39\%) as resolved. The mean age and refractive error for each subtype is summarized in Table 1.

\section{Angiographic data}

All patients with CSC were examined using simultaneous FA and IA. FA showed leakage from the RPE in 25 of 27 (93\%) active eyes. Early-phase IA was studied in 1 eye of each patient, and the area with filling delay in choroidal arteries and choriocapillaris was seen in 20 of 28 eyes (71\%). In late-phase IA, choroidal vascular hyperpermeability was seen in all 44 eyes (100\%), and punctuate hyperfluorescent spots were seen in 40 of 44 eyes (91\%). In active eyes, choroidal filling delay was observed in 13 of 18 eyes (72\%), choroidal hyperpermeability in all 27 eyes (100\%), and punctuate hyperfluorescent spots in 25 of 27 eyes (93\%). In resolved eyes, choroidal filling delay was observed in 7 of 10 eyes (70\%), choroidal hyperpermeability in all 17 eyes (100\%), and punctuate hyperfluorescent spots in 15 of 17 eyes $(88 \%)$.

\section{Macular choroidal thickness in ETDRS sectors}

The mean choroidal thickness of each ETDRS sector was significantly greater in eyes with CSC compared with normal eyes (Figs 1 and 2, Table 2). Whole macular choroidal thickness in eyes with CSC was $329.3 \pm 83.0 \mu \mathrm{m}$, which was greater than that in normal eyes $(233.0 \pm 67.0 \mu \mathrm{m})(P<0.001$, by $t$-test $)$.

In unilateral cases $(n=19)$, macular choroidal thickness was greater in 8 of 9 sectors in affected eyes than in unaffected fellow eyes (Table 3). The whole macular choroidal thickness was greater in eyes with unilateral CSC $(326.6 \pm 98.4 \mu \mathrm{m})$ than in unaffected fellow eyes $(254.2 \pm 86.8 \mu \mathrm{m})(P=0.021$, by $t$-test $)$. 
The whole macular choroidal thickness did not differ between unaffected fellow eyes ( $\mathrm{n}$ $=19 ; 254.2 \pm 86.8 \mu \mathrm{m})$ and the normal eyes $(233.0 \pm 67.0 \mu \mathrm{m})(P=0.423$, by $t$-test $)$. The whole macular choroidal thickness of the unaffected fellow eyes with choroidal hyperpermeability $(n=11 ; 273.4 \pm 86.6 \mu \mathrm{m})$ was slightly increased compared with eyes without choroidal hyperpermeability $(n=8 ; 227.6 \pm 85.3 \mu \mathrm{m})$ or normal eyes $(n=17$; $233.0 \pm 67.0 \mu \mathrm{m})$, but the difference was not significant $(P=0.268$ and $P=0.176$, respectively, by $t$-test).

There was no significant difference between choroidal thickness in active eyes and that for resolved eyes in each ETDRS sector (Supplemental Table 1). The mean whole macular choroidal thickness was $342.2 \pm 75.9 \mu \mathrm{m}$ in active eyes, and $308.8 \pm 91.7 \mu \mathrm{m}$ in resolved eyes $(P=0.196$ by $t$-test).

The mean whole macular choroidal thickness was rather more in eyes $(n=7)$ that had undergone laser photocoagulation than in eyes $(n=37)$ that had not $(395.0 \pm 74.1 \mu \mathrm{m}$ and $316.8 \pm 79.4 \mu \mathrm{m}$, respectively; $P=0.020$, by $t$-test)

Mean choroidal thickness of each area in the ETDRS showed no significant difference among classic CSC, chronic CSC, and MPPE; however, all of them showed increased thickness compared with normal eyes (Figs. 1 and 2, Table 4). The mean whole macular choroidal thickness was $326.9 \pm 83.1 \mu \mathrm{m}$ in cases of classic CSC, $325.4 \pm 93.3$ $\mu \mathrm{m}$ in chronic CSC, and $359.0 \pm 15.5 \mu \mathrm{m}$ in MPPE, which were greater than that of normal eyes $(P<0.001, P=0.002, P=0.002$, respectively, by $t$-test).

In active eyes, the mean choroidal thickness of each ETDRS sector showed no significant difference between classic CSC, chronic CSC, and MPPE. The mean whole macular choroidal thickness was $341.9 \pm 72.9 \mu \mathrm{m}$ in classic CSC, $339.0 \pm 99.7 \mu \mathrm{m}$ in 
chronic CSC, and $352.0 \pm 8.0 \mu \mathrm{m}$ in MPPE. In resolved eyes, the mean choroidal thickness of each ETDRS sector showed no significant difference between classic CSC and chronic CSC. The mean whole macular choroidal thickness was $292.7 \pm 100.1 \mu \mathrm{m}$ for classic CSC and $313.4 \pm 91.2 \mu \mathrm{m}$ for chronic CSC.

A significant difference was found in the refractive error between eyes with classic CSC, chronic CSC, and MPPE. Therefore, we only analyzed CSC eyes with a refractive error of \pm 1.5 diopters. The mean choroidal thickness of each ETDRS sector showed no significant differences between the 3 disease subtypes (Supplemental Table 2).

\section{Choroidal thickness and angiographic changes}

The relationship between the mean choroidal thickness in each $5 \times 5$ grid sector and the angiographic findings were evaluated (Fig. 3 and 4; Table 5). Sectors with angiographic abnormalities such as choroidal filling delay and hyperpermeability were defined as those that involved more than $50 \%$ of the sector area. Choroidal thickness was greater in areas with leakage on FA $(361.9 \pm 81.5 \mu \mathrm{m})$ than in areas without leakage $(337.6 \pm$ $81.5 \mu \mathrm{m})(P=0.001$, by $t$-test $)$. Choroidal thickness was greater in areas with choroidal vascular hyperpermeability on IA $(356.8 \pm 92.7 \mu \mathrm{m})$ than in unaffected areas $(319.4 \pm$ $90.7 \mu \mathrm{m})(P<0.001$, by $t$-test $)$. In addition, choroidal thickness was greater in areas with punctate hyperfluorescent spots on IA $(367.6 \pm 87.9 \mu \mathrm{m})$ than in unaffected areas $(333.8 \pm 87.9 \mu \mathrm{m})(P<0.001$, by $t$-test $)$. However, choroidal thickness did not differ between areas with choroidal filling delay and areas without it ( $P=0.872$, by $t$-test).

Correlation between age, refractive error, and choroidal thickness in eyes with CSC 
Supplemental Table 3 shows Pearson's correlation coefficient and partial correlation coefficient between macular choroidal thickness and age or refractive error. The whole macular choroidal thickness was correlated significantly with age after adjusting for refractive error $(r=-0.477 ; P=0.001)$, and with refractive error after adjusting for age $(r$ $=0.366 ; P=0.016)$.

\section{Discussion}

Although the pathophysiology of CSC remains unknown, there is strong angiographic evidence that the key underlying mechanism involves choroidal vascular abnormalities. Studies using IA have shown a variety of choroidal abnormalities in CSC patients, including variable filling delay in the regions within the choroid, ${ }^{(2-6)}$ choroidal venous dilation, ${ }^{(2,4-5)}$ multiple areas of choroidal vascular hyperpermeability, ${ }^{(1-17)}$ and punctate choroidal hyperfluorescent spots, ${ }^{(14)}$ suggesting that a generalized choroidal vascular disturbance occurs in CSC. Other studies have shown evidence of hyperdynamic circulation within the choroid in eyes with CSC. ${ }^{(37,38)}$

Recently, Imamura et al. reported that EDI-OCT demonstrated the presence of a very thick choroid in patients with CSC. ${ }^{(18)}$ On the other hand, Maruko et al. reported using EDI-OCT that focal laser photocoagulation did not result in any change in choroidal thickness, whereas PDT led to choroidal thinning and decreased hyperpermeability seen during IA in eyes with CSC. ${ }^{(19)}$ However, in the studies using EDI-OCT, choroidal thickness measurements were indicated by a representative value obtained at the foveal center. This measurement tends to be influenced by focal thickening or thinning of the choroid, or more often with irregularity of the inner chorioscleral border. In the 
current study, using 3D-raster scanning images obtained by high-penetrating SS-OCT, eyes with CSC were found to have thickened choroids in the whole macular area and focally increased choroidal thickness associated with angiographic findings.

Several investigators have reported that foveal choroidal thickness is correlated with age, axial length, or refractive error in normal subjects. ${ }^{(16,34,39)}$ We also found that the mean macular choroidal thickness had a negative correlation with age and refractive error in eyes with CSC. These results suggest that both age and refractive error should be considered in measuring choroidal thickness even in pathologic eyes. In the current study, age and refractive error-matched normal controls were used, and explorative analysis was also performed when no difference was found in age or refractive error in eyes with CSC subtypes.

Central macular choroidal thickness in eyes with CSC was greater than that in normal eyes, which is consistent with the results of Imamura et al. ${ }^{(18)}$ and Maruko et al. ${ }^{(20)}$ In addition, choroidal thickness in the whole macular area and in each ETDRS-sector area was found to be increased in eyes with CSC. This result corresponds to the finding that choroidal abnormalities on IA are not only confined to the fovea, but also extend into other areas of the macula. Moreover, the central choroidal thickness in unilateral symptomatic eyes was greater than the unaffected fellow eyes in most of ETDRS sectors. Maruko et al. ${ }^{(20)}$ reported that the subfoveal choroidal thickness in unilateral symptomatic eyes was greater than that in fellow eyes, which is consistent with our study. They also showed that subfoveal choroidal thickness was greater in unaffected fellow eyes than in age-matched normal eyes, and greater in unaffected eyes with choroidal vascular hyperpermeability than in unaffected eyes without choroidal vascular 
hyperpermeabilty. Our subjects showed a similar tendency, but the results were not statistically significant, probably because of the small sample size.

There was no significant difference in the choroidal thickness between active eyes and resolved eyes. These findings suggest that choroidal thickness is increased in patients with CSC regardless of the resolution of serous retinal detachment. In addition, lida et al. ${ }^{(5)}$ found that choroidal vascular abnormalities in IA persist in both eyes even after the cessation of leakage from the RPE. Thus, choroidal structural abnormalities may persist even after the resolution of serous retinal detachment. These findings may have a relationship with the well-known phenomenon that serous retinal detachment tends to recur, especially in areas with choroidal hyperpermeability, in patients with CSC. ${ }^{(5,10-11)}$ Maruko et al reported that, in a longitudinal study, focal laser photocoagulation did not result in any change in choroidal thickness although serous retinal detachment was decreased. ${ }^{(19)}$ In the current cross-sectional study, the mean choroidal thickness of eyes with a history of laser photocoagulation was rather greater than that of eyes without photocoagulation treatment. Taken together, it seems that laser photocoagulation therapy does not result in a decrease in choroidal thickness, but just covers the leakage from RPE.

Some patients with CSC have shown recent onset of the disease with 1 or only a few specific points of leakage from the RPE; these eyes are commonly termed as having classic CSC. ${ }^{(19)}$ Other patients with chronic disease have broad areas of granular hyperfluorescence during FA, associated with many indistinct areas of leakage. These patients have chronic CSC, which has also been called diffuse retinal pigment epitheliopathy. ${ }^{(8,13,19)}$ A type of exudative retinal detachment, usually manifested with flat 
serous retinal detachment in the posterior pole and bullous retinal detachment in the lower periphery, was reported as bullous retinal detachment in $1973,{ }^{(36)}$ and termed as MPPE in 1999. ${ }^{(35)}$ MPPE has been considered as an unusual manifestation and severe form of CSC. ${ }^{(35)}$ In the current study, all eyes of these types showed choroidal hyperpermeability, and choroidal thickness was equally increased in classic CSC, chronic CSC, and MPPE. The underlying IA abnormality unifying them is choroidal vascular hyperpermeability, and the underlying OCT finding unifying them is increased choroidal thickness. Thus, classic CSC, chronic CSC, and MPPE may share a common pathology with choroidal vascular abnormalities, although the visual prognosis is different.

Choroidal thickness was greater in the area with leakage points on FA than in the area without it. Moreover, choroidal thickness was greater in the area with choroidal hyperpermeability on IA than in the unaffected areas. Increase in hydrostatic pressure within the choroid sufficient to cause changes to the overlying RPE and retina may cause structural changes within the choroid itself. Focal thickening of the choroid in these areas may suggest that focal hydrostatic pressure within the choroid is increased in the area with choroidal vascular hyperpermeability, resulting in pigment epithelial detachment with subsequent RPE defect, ${ }^{(40)}$ which in turn results in focal leakage into the subretinal space. In addition, choroidal thickness was greater in areas with punctate hyperfluorescent spots on IA than in unaffected areas. Punctate hyperfluorescent spots have been reported to be located in the inner choroid, and seen at the center of the focal hyperfluorescent area on IA. This area often expands from the punctate spots, ${ }^{(14)}$ possibly resulting in increased pressure in the inner choroid and/or Bruch's membrane and leading to a break in the barrier of RPE. Thus, focal increased choroidal thickness 
in the area with punctate lesions may be explained by increased pressure in the inner choroid.

There are some limitations to this study. Although the high-penetrating SS-OCT increases the sensitivity of the choroid, scattering of light by the RPE and choroid still occurs, which makes visualization of the chorio-scleral interface difficult in some patients. There is currently no automated segmentation software available for measuring choroidal thickness, thus we performed all segmentations manually. However, we have previously shown good interobserver repeatability with this technique ${ }^{(34)}$ Despite these limitations, we demonstrated that choroidal thickness was increased in the whole macular area in all types of CSC, and that choroidal thickness was associated with leakage from the RPE, choroidal vascular hyperpermeability, and punctate hyperfluorescent lesions. These findings provide additional evidence that CSC may be caused by increased hydrostatic pressure in the choroid. 


\section{References}

1. Guyer DR, Yannuzzi LA, Slankter JS, et al. Digital indocyanine green angiography of central serous chorioretinopathy. Arch Ophthalmol 1994:112:1057-62.

2. Menchini U, Virgili G, Lanzentte $P$, Ferrari E. Indocyanine green angiography in central serous chorioretinopathy. Int Ophtalmol 1997;21:57-69.

3. Prunte $\mathrm{C}$. Indocyanine green angiographic findings in central serous chorioretinopathy. Int Ophthalmol 1995:19:77-82.

4. Prunte C, Flammer J. Choroidal capillary and venous congestion in central serous chorioretinopathy. Am J Ophthalmol 1996;121:26-34.

5. lida T, Kishi S, Hagimura N, et al. Persistence and bilateral choroidal vascular abnormality in central serous chorioretinopathy. Retina 1999;19:508-12.

6. Kitaya N, Nagaoka T, Hikichi T, et al. Features of abnormal choroidal circulation in central serous chorioretinopathy. Br J Ophthalmol 2003;87:709-12.

7. Spaide RF, Goldbaum M, Wong DW, et al. Serous detachment of the retina. Retina 2003;23:820-46.

8. Spaide RF, Hall L, Haas A, et al. Indocyanine green videoangiography of older patients with central serous chorioretinopathy. Retina 1996;16:203-13.

9. Spaide RF, Campeas L, Haas A, et al. Central serous chorioretinopathy in younger and older adults. Ophthalmology 1996;103:2070-9.

10. Piccolino FC, Borgia L. Central serous chorioretinopathy and indocyanine green angiography. Retina 1994;14:231-42.

11. Piccolino FC, Borgia L, Zinicola E, Zingirian M. Indocyanine green angiographic 
findings in central serous chorioretinopathy. Eye 1995;9:324-32.

12. Scheider A, Nasemann JE, Lund OE. Fluorescein and indocyanine green angiographies of central serous choroidopathy by scanning laser ophthalmoscopy. Am J Ophthalmol 1993;115:50-6.

13. Klais CM, Ober MD, Ciardella AP, Yannuzzi LA. Central serous chorioretinopathy. In: S.J. Ryan, D.R. Hinton, A.P. Schachat and C.P. Wilkinson, eds. Retina. Vol. $2.4^{\text {th }}$ ed. Mosby, Philadelphia: Elsevier; 2006:1135-61.

14. Tsujikawa A, Ojima $Y$, Yamashiro K, et al. Punctate hyperfluorescent spots associated with central serous chorioretinopathy as seen on indocyanine green angiography. Retina 2010;30:801-9.

15. Spaide RF, Koizumi H, Pozonni MC. Enhanced depth imaging spectral-domain optical coherence tomography. Am J Ophthalmol 2008;146:496-500.

16. Margolis R, Spaide RF. Pilot study of enhanced depth imaging optical coherence of tomography of the choroid in normal eyes. Am J Ophthalmol 2009;147:811-5.

17. Doro D, Visentin S, Maimone PE, et al. High-resolution ultrasonography in central serous chorioretinopathy. Am J Ophthalmol 2005;139:550-2.

18. Imamura Y, Fujiwara, T, Margolis R, Spaide RF. Enhanced depth imaging optical coherence tomography of the choroid in central serous chorioretinopathy. Retina 2009;29:1469-73.

19. Maruko I, lida T, Sugano Y, et al. Subfoveal choroidal thickness after treatment of central serous chorioretinopathy. Ophthalmol 2010;117:1792-99. 
20. Maruko I, lida T, Sugano Y, et al. Subfoveal choroidal thickness in fellow eyes of patients with central serous chorioretinopathy. Retina. In Press.

21. Spaide RF. Enhanced depth imaging optical coherence tomography of retinal pigment epithelial detachment in age-related macular degeneration. Am J Ophthalmol $2009 ; 147: 644-52$

22. Reibaldi M, Boscia F, Avitabile T, et al. Enhanced depth imaging optical coherence tomography of choroid in idiopathic macular hole: A cross-sectional prospective study. Am J Ophthalmol 2011;151:112-7.

23. Chung SE, Kang SW, Lee JH, Kim YT. Choroidal thickness in polypoidal choroidal vasculopathy and exudative age-related macular degeneration. Ophthalmology 2011;118:840-5

24. Maruko I, lida T, Sugano Y, et al. Subfoveal choroidal thickness after treatment of Vogt-Koyanagi-Harada disease. Retina 2011;31:510-7.

25. Maruko I, lida T, Sugano Y, et al. Subfoveal retinal and choroidal thickness after verteporfin photodynamic therapy for polypoidal choroidal vasculopathy. Am J Ophthalmol 2011;151:594-603.

26. Esmaeelpour M, Povazay B, Hermann B, et al. Three-dimensional 1060-nm OCT: choroidal thickness maps in normal subjects and improved posterior segment visualization in cataract patients. Invest Ophthalmol Vis Sci 2010;51:5260-6. 27. Povazay B, Bizheva K, Hermann B, et al. Enhanced visualization of choroidal vessels using ultrahigh resolution ophthalmic OCT at $1050 \mathrm{~nm}$. Opt Express 2003;11:1980-6. 
28. Huber R, Adler DC, Srinivasan VJ, Fujimoto JG. Fourier domain mode locking at $1050 \mathrm{~nm}$ for ultra-high-speed optical coherence tomography of the human retina at 236,000 axial scans per second. Opt Lett 2007;32:2049-51.

29. de Bruin DM, Burnes DL, Loewenstein J, et al. In vivo three-dimensional imaging of neovascular age-related macular degeneration using optical frequency domain imaging at $1050 \mathrm{~nm}$. Invest Ophthalmol Vis Sci 2008;49:4545-52.

30. Potsaid B, Baumann B, Huang D, et al. Ultrahigh speed 1050nm swept source/Fourier domain OCT retinal and anterior segment imaging at 100,000 to 400,000 axial scans per second. Opt Express 2010;18:20029-48.

31. Srinivasan VJ, Huber R, Gorczynska I, et al. High-speed, high-resolution optical coherence tomography retinal imaging with a frequency-swept laser at $850 \mathrm{~nm}$. Opt Lett 2007;32:361-3.

32. Unterhuber A, Povazay B, Hermann B, et al. In vivo retinal optical coherence tomography at $1040 \mathrm{~nm}$ - enhanced penetration into the choroid. Opt Express 2005;13:3252-8.

33. Yasuno $\mathrm{Y}$, Hong $\mathrm{Y}$, Makita S, et al. In vivo high-contrast imaging of deep posterior eye by 1-microm swept source optical coherence tomography and scattering optical coherence angiography. Opt Express 2007;15:6121-39.

34. Hirata M, Tsujikawa A, Matsumoto A, et al. Macular choroidal thickness and volume in normal subjects measured by swept-source optical coherence tomography. Invest Ophthalmol Vis Sci 2011:52:4971-8. 
35. Uyama M, Matsunaga $\mathrm{H}$, Matsubara $\mathrm{T}$, et al. Indocyanine green angiography and pathophysiology of multiple posterior pigment epitheliopathy. Retina 1999;19:12-21.

36. Gass JD. Bullous retinal detachment. An unusual manifestation of idiopathic central serous choroidopathy. Am J Ophthalmol 1973;75:810-21.

37. Tittl M, Polska E, Kircher K, et al. Topical fundus pulsation measurement in patients with active central serous chorioretinopathy. Arch Ophthalmol 2003;121:975-8.

38. Tittl M, Maar N, Polska E, et al. Choroidal hemodynamic changes during isometric exercise in patients with inactive central serous chorioretinopathy. Invest Ophthalmol Vis Sci 2005;46:4717-21.

39. Ikuno Y, Kawaguchi, Nouchi T, Yasuno Y. Choroidal thickness in healthy Japanese subjects. Invest Ophthalmol Vis Sci 2010;51:2173-6.

40. Fujimoto H, Gomi F, Wakabayashi T, et al. Morphologic changes in acute central serous chorioretinopathy evaluated by fourier-domain optical coherence tomography. Ophthalmology 2008;115:1494-500. 
Table 1. Mean age and refractive error in eyes with each CSC subtype

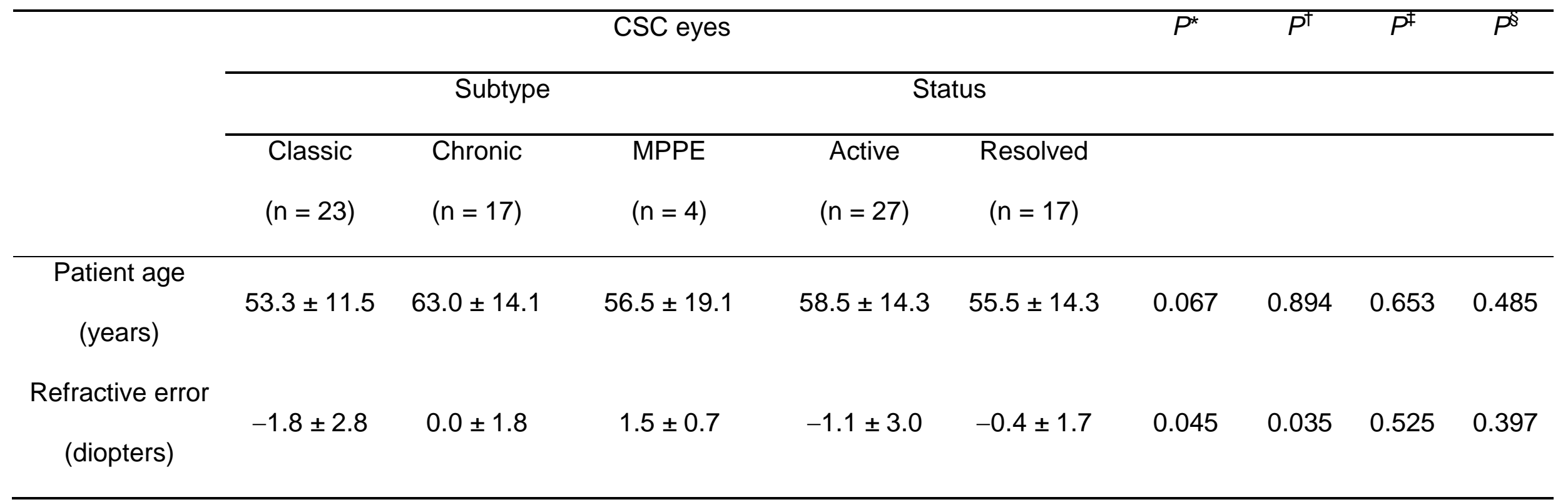

Values are represented as mean \pm standard deviation.

CSC, central serous chorioretinopathy; MPPE, multifocal posterior pigment epitheliopathy

${ }^{*}$ Comparison between classic CSC and chronic CSC by the Turkey-Kramer test

${ }^{\dagger}$ Comparison between classic CSC and MPPE by the Turkey-Kramer test

${ }^{\ddagger}$ Comparison between chronic CSC and MPPE by the Turkey-Kramer test

$\S$ Comparison between active and resolved CSC by $t$-test 
Table 2. Mean choroidal thickness in eyes with CSC and in normal eyes

\begin{tabular}{cccc}
\hline Area $(\mu \mathrm{m})$ & CSC & Normal eyes & $P^{\star}$ \\
& $(\mathrm{n}=44)$ & $(\mathrm{n}=17)$ & $<0.001$ \\
\hline Whole macula & $329.3 \pm 83.0$ & $233.0 \pm 67.0$ & $<0.001$ \\
Center & $374.3 \pm 92.9$ & $248.4 \pm 77.4$ & $<0.001$ \\
Inner temporal & $366.5 \pm 91.5$ & $247.4 \pm 76.8$ & $<0.001$ \\
Inner superior & $363.2 \pm 92.9$ & $250.4 \pm 73.4$ & $<0.001$ \\
Inner nasal & $352.7 \pm 96.1$ & $229.6 \pm 77.6$ & $<0.001$ \\
Inner inferior & $360.1 \pm 93.4$ & $246.3 \pm 72.7$ & $<0.001$ \\
Outer temporal & $327.2 \pm 83.0$ & $237.6 \pm 72.3$ & 0.004 \\
Outer superior & $333.5 \pm 88.0$ & $261.9 \pm 67.2$ & $<0.001$ \\
Outer nasal & $285.7 \pm 91.3$ & $189.0 \pm 70.3$ & $<0.001$ \\
Outer inferior & $326.9 \pm 82.7$ & $228.8 \pm 68.6$ &
\end{tabular}

Values are represented as mean \pm standard deviation.

CSC, central serous chorioretinopathy

Inner, 1-3 mm from foveal center; Outer, 3-6 $\mathrm{mm}$ from the foveal center. 
* Comparison between CSC and normal eyes by $t$-test 
Table 3. Mean choroidal thickness in eyes with unilateral CSC and in unaffected fellow eyes

\begin{tabular}{|c|c|c|c|}
\hline \multirow{3}{*}{ Area $(\mu \mathrm{m})$} & Unilateral & Unaffected & \multirow{3}{*}{$P^{\star}$} \\
\hline & CSC eyes & fellow eyes & \\
\hline & $(n=19)$ & $(n=19)$ & \\
\hline Whole macula & $326.6 \pm 98.4$ & $254.2 \pm 86.8$ & 0.021 \\
\hline Center & $376.5 \pm 115.2$ & $287.6 \pm 101.6$ & 0.016 \\
\hline Inner temporal & $373.0 \pm 116.8$ & $286.0 \pm 98.3$ & 0.018 \\
\hline Inner superior & $353.7 \pm 110.7$ & $275.8 \pm 89.4$ & 0.022 \\
\hline Inner nasal & $348.3 \pm 112.8$ & $260.4 \pm 98.0$ & 0.015 \\
\hline Inner inferior & $368.5 \pm 112.2$ & $269.0 \pm 100.8$ & 0.007 \\
\hline Outer temporal & $330.5 \pm 104.6$ & $263.2 \pm 86.7$ & 0.038 \\
\hline Outer superior & $322.3 \pm 96.8$ & $265.1 \pm 84.5$ & 0.060 \\
\hline Outer nasal & $275.3 \pm 108.1$ & $206.9 \pm 89.0$ & 0.040 \\
\hline
\end{tabular}



Table 4 Mean choroidal thickness in each subtype of central serous chorioretinopathy vs normal eyes

\begin{tabular}{|c|c|c|c|c|c|c|c|}
\hline \multirow[t]{2}{*}{ Area $(\mu \mathrm{m})$} & \multicolumn{3}{|c|}{ CSC subtype } & \multirow[t]{2}{*}{ normal eyes $(n=17)$} & \multirow{2}{*}{$\begin{array}{c}P \text {-value* } \\
\text { (t-test) }\end{array}$} & \multirow{2}{*}{$\begin{array}{c}P \text {-value }{ }^{\dagger} \\
\text { (t-test) }\end{array}$} & \multirow{2}{*}{$\begin{array}{c}P \text {-value } \\
\text { (t-test) }\end{array}$} \\
\hline & Classic $(n=23)$ & Chronic $(n=17)$ & MPPE $(n=4)$ & & & & \\
\hline Whole macula & $326.9 \pm 83.1$ & $325.4 \pm 93.3$ & $359.0 \pm 15.5$ & $233.0 \pm 67.0$ & $<0.001$ & 0.002 & 0.002 \\
\hline Center & $374.2 \pm 94.9$ & $368.7 \pm 103.0$ & $398.7 \pm 17.1$ & $248.4 \pm 77.4$ & $<0.001$ & 0.001 & 0.001 \\
\hline Inner temporal & $365.0 \pm 94.8$ & $368.2 \pm 100.4$ & $367.9 \pm 17.6$ & $247.4 \pm 76.8$ & $<0.001$ & $<0.001$ & 0.006 \\
\hline Inner superior & $360.5 \pm 96.0$ & $362.2 \pm 101.9$ & $382.6 \pm 16.9$ & $250.4 \pm 73.4$ & $<0.001$ & 0.001 & 0.002 \\
\hline Inner nasal & $346.7 \pm 94.0$ & $350.3 \pm 110.0$ & $396.9 \pm 15.9$ & $229.6 \pm 77.6$ & $<0.001$ & 0.001 & $<0.001$ \\
\hline Inner inferior & $354.1 \pm 90.7$ & $361.6 \pm 108.8$ & $387.7 \pm 17.3$ & $246.3 \pm 72.7$ & $<0.001$ & 0.001 & 0.001 \\
\hline Outer temporal & $327.1 \pm 86.6$ & $326.5 \pm 90.3$ & $331.2 \pm 16.9$ & $237.6 \pm 72.3$ & 0.001 & 0.003 & 0.021 \\
\hline Outer superior & $338.9 \pm 89.3$ & $317.7 \pm 95.6$ & $369.6 \pm 17.5$ & $261.9 \pm 67.2$ & 0.005 & 0.058 & 0.006 \\
\hline Outer nasal & $277.2 \pm 89.7$ & $283.2 \pm 101.3$ & $344.9 \pm 23.6$ & $189.0 \pm 70.3$ & 0.002 & 0.004 & $<0.001$ \\
\hline Outer inferior & $322 . \pm 80.8$ & $326.3 \pm 95.0$ & $355.2 \pm 25.9$ & $228.8 \pm 68.6$ & $<0.001$ & 0.002 & 0.002 \\
\hline
\end{tabular}

Values are mean \pm standard deviation

CSC, central serous chorioretinopathy, MPPE=multifocal posterior pigment epitheliopathy 
Inner = 1-3 mm from foveal center; Outer = 3-6 $\mathrm{mm}$ from central fovea.

* Comparison between classic CSC and normal eyes by $t$-test

${ }^{\dagger}$ Comparison between chronic CSC and normal eyes by $t$-test

‡ Comparison between MPPE and normal eyes by $t$-test 
Table 5. Mean choroidal thickness in areas with angiographic abnormalities versus areas without angiographic abnormalities

\begin{tabular}{cccc}
\hline Area $(\mu \mathrm{m})$ & Affected area & Unaffected area & $P^{\star}$ \\
\hline FA leakage & $361.9 \pm 81.5$ & $337.6 \pm 81.5$ & 0.001 \\
Choroidal filling delay & $339.1 \pm 100.3$ & $340.6 \pm 93.9$ & 0.872 \\
Choroidal & $356.8 \pm 92.7$ & $319.4 \pm 90.7$ & $<0.001$ \\
hyperpermeability & & & \\
Punctuate & & & \\
hyperfluorescent & $367.6 \pm 87.9$ & $333.8 \pm 87.9$ & $<0.001$ \\
spots & & & \\
\hline
\end{tabular}

Values are represented as mean \pm standard deviation.

FA, fluorescence angiography

${ }^{*}$ Comparison between affected and unaffected area by $t$-test 
Supplemental Table 1. Mean choroidal thickness in active and resolved eyes

\begin{tabular}{cccc}
\hline Area $(\mu \mathrm{m})$ & Active eyes & Resolved eyes & $P^{\star}$ \\
\hline Whole macula & $342.2 \pm 75.9$ & $(\mathrm{n}=17)$ & \\
Center & $386.8 \pm 89.9$ & $354.4 \pm 96.9$ & 0.197 \\
Inner temporal & $375.9 \pm 86.2$ & $351.5 \pm 100.2$ & 0.265 \\
Inner superior & $371.9 \pm 92.1$ & $349.2 \pm 95.4$ & 0.395 \\
Inner nasal & $370.0 \pm 93.2$ & $325.1 \pm 97.0$ & 0.437 \\
Inner inferior & $375.0 \pm 85.1$ & $336.3 \pm 103.5$ & 0.133 \\
Outer temporal & $334.2 \pm 74.4$ & $316.3 \pm 96.5$ & 0.184 \\
Outer superior & $344.5 \pm 84.2$ & $316.0 \pm 93.5$ & 0.493 \\
Outer nasal & $302.7 \pm 84.2$ & $258.6 \pm 98.2$ & 0.300 \\
Outer inferior & $343.9 \pm 66.4$ & $299.8 \pm 99.7$ & 0.120 \\
\hline Values are represented & & & \\
\hline & & & \\
\hline
\end{tabular}

Values are represented as mean \pm standard deviation.

CSC, central serous chorioretinopathy

Inner, 1-3 mm from the foveal center; Outer, 3-6 $\mathrm{mm}$ from the central fovea

* Comparison between active and resolved eyes by $t$-test 
Supplemental Table 2. Mean choroidal thickness in eyes of each CSC subtype with refractive error of \pm 1.5 diopters

\begin{tabular}{|c|c|c|c|c|c|c|}
\hline \multirow[t]{3}{*}{ Area $(\mu \mathrm{m})$} & \multicolumn{3}{|c|}{ CSC eyes } & \multirow[t]{3}{*}{$P^{\star}$} & \multirow[t]{3}{*}{$P^{\dagger}$} & \multirow[t]{3}{*}{$P^{\ddagger}$} \\
\hline & Classic & Chronic & MPPE & & & \\
\hline & $(n=15)$ & $(n=10)$ & $(n=2)$ & & & \\
\hline Whole macular & $352.2 \pm 85.1$ & $342.5 \pm 107.3$ & $361.5 \pm 26.4$ & 0.964 & 0.990 & 0.962 \\
\hline Center & $405.7 \pm 92.6$ & $379.7 \pm 115.2$ & $392.6 \pm 23.2$ & 0.802 & 0.983 & 0.985 \\
\hline Inner temporal & $393.5 \pm 97.9$ & $374.1 \pm 119.6$ & $363.3 \pm 25.3$ & 0.893 & 0.922 & 0.990 \\
\hline Inner superior & $389.0 \pm 99.1$ & $378.4 \pm 125.7$ & $384.9 \pm 18.5$ & 0.969 & 0.999 & 0.997 \\
\hline Inner nasal & $377.9 \pm 92.8$ & $364.9 \pm 120.7$ & $392.3 \pm 25.4$ & 0.949 & 0.981 & 0.937 \\
\hline Inner inferior & $384.9 \pm 88.3$ & $377.5 \pm 125.2$ & $376.6 \pm 19.1$ & 0.982 & 0.993 & 1.000 \\
\hline Outer temporal & $350.4 \pm 92.0$ & $337.6 \pm 110.9$ & $333.5 \pm 26.1$ & 0.945 & 0.971 & 0.998 \\
\hline Outer superior & $366.1 \pm 93.0$ & $341.3 \pm 112.7$ & $370.6 \pm 23.6$ & 0.814 & 0.998 & 0.923 \\
\hline Outer nasal & $303.1 \pm 94.9$ & $341.3 \pm 112.7$ & $370.6 \pm 23.6$ & 0.976 & 0.812 & 0.760 \\
\hline Outer inferior & $341.0 \pm 81.9$ & $354.1 \pm 98.2$ & $366.9 \pm 25.2$ & 0.928 & 0.918 & 0.981 \\
\hline
\end{tabular}

Values are represented as mean \pm standard deviation.

CSC, central serous chorioretinopathy; D, diopter; MPPE, multifocal posterior pigment epitheliopathy

Inner, 1-3 mm from the foveal center; Outer, 3-6 $\mathrm{mm}$ from the central fovea

* Comparison between classic CSC and chronic CSC by the Turkey-Kramer test

${ }^{\dagger}$ Comparison between classic CSC and MPPE by the Turkey-Kramer test 
${ }^{\ddagger}$ Comparison between chronic CSC and MPPE by the Turkey-Kramer test 
Supplemental Table 3. Pearson's correlation and partial correlation coefficient between choroidal thickness and age/refractive error

\begin{tabular}{|c|c|c|c|c|c|c|c|c|}
\hline \multirow[t]{2}{*}{ Area $(\mu \mathrm{m})$} & \multicolumn{2}{|l|}{ Age } & \multicolumn{2}{|c|}{ Adjusting for refractive error } & \multicolumn{2}{|c|}{ Refractive error } & \multicolumn{2}{|c|}{ Adjusting for age } \\
\hline & $\begin{array}{l}\text { R (Pearson's } \\
\text { correlation } \\
\text { coefficient) }\end{array}$ & $P$ & $\begin{array}{l}\text { R (Partial } \\
\text { correlation } \\
\text { coefficient) }\end{array}$ & $P$ & $\begin{array}{l}\text { R (Pearson's } \\
\text { correlation } \\
\text { coefficient) }\end{array}$ & $P$ & $\begin{array}{l}\mathrm{R} \text { (Partial } \\
\text { correlation } \\
\text { coefficient) }\end{array}$ & $P$ \\
\hline Whole macular & -0.401 & 0.007 & -0.477 & 0.001 & 0.244 & 0.111 & 0.366 & 0.016 \\
\hline Center & -0.373 & 0.013 & -0.461 & 0.002 & 0.281 & 0.064 & 0.396 & 0.009 \\
\hline Inner temporal & -0.383 & 0.010 & -0.447 & 0.003 & 0.211 & 0.169 & 0.323 & 0.035 \\
\hline Inner superior & -0.463 & 0.003 & -0.482 & 0.001 & 0.146 & 0.345 & 0.269 & 0.081 \\
\hline Inner nasal & -0.318 & 0.035 & -0.417 & 0.005 & 0.325 & 0.031 & 0.423 & 0.005 \\
\hline Inner inferior & -0.285 & 0.061 & -0.390 & 0.010 & 0.346 & 0.022 & 0.433 & 0.004 \\
\hline Outer temporal & -0.456 & 0.002 & -0.508 & 0.001 & 0.164 & 0.289 & 0.298 & 0.053 \\
\hline Outer superior & -0.502 & 0.001 & -0.549 & $<0.001$ & 0.141 & 0.361 & 0.291 & 0.058 \\
\hline
\end{tabular}




$\begin{array}{lllllllll}\text { Outer nasal } & -0.258 & 0.091 & -0.354 & 0.020 & 0.330 & 0.029 & 0.406 & 0.007 \\ \text { Outer inferior } & -0.331 & 0.028 & -0.400 & 0.008 & 0.234 & 0.126 & 0.329 \\ \end{array}$




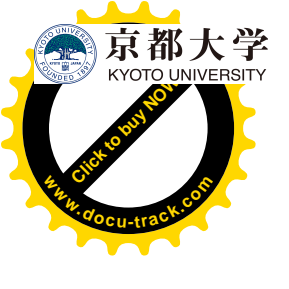


$F=$ 京都大学

A Self-archived copy in
insity Research Information Repository
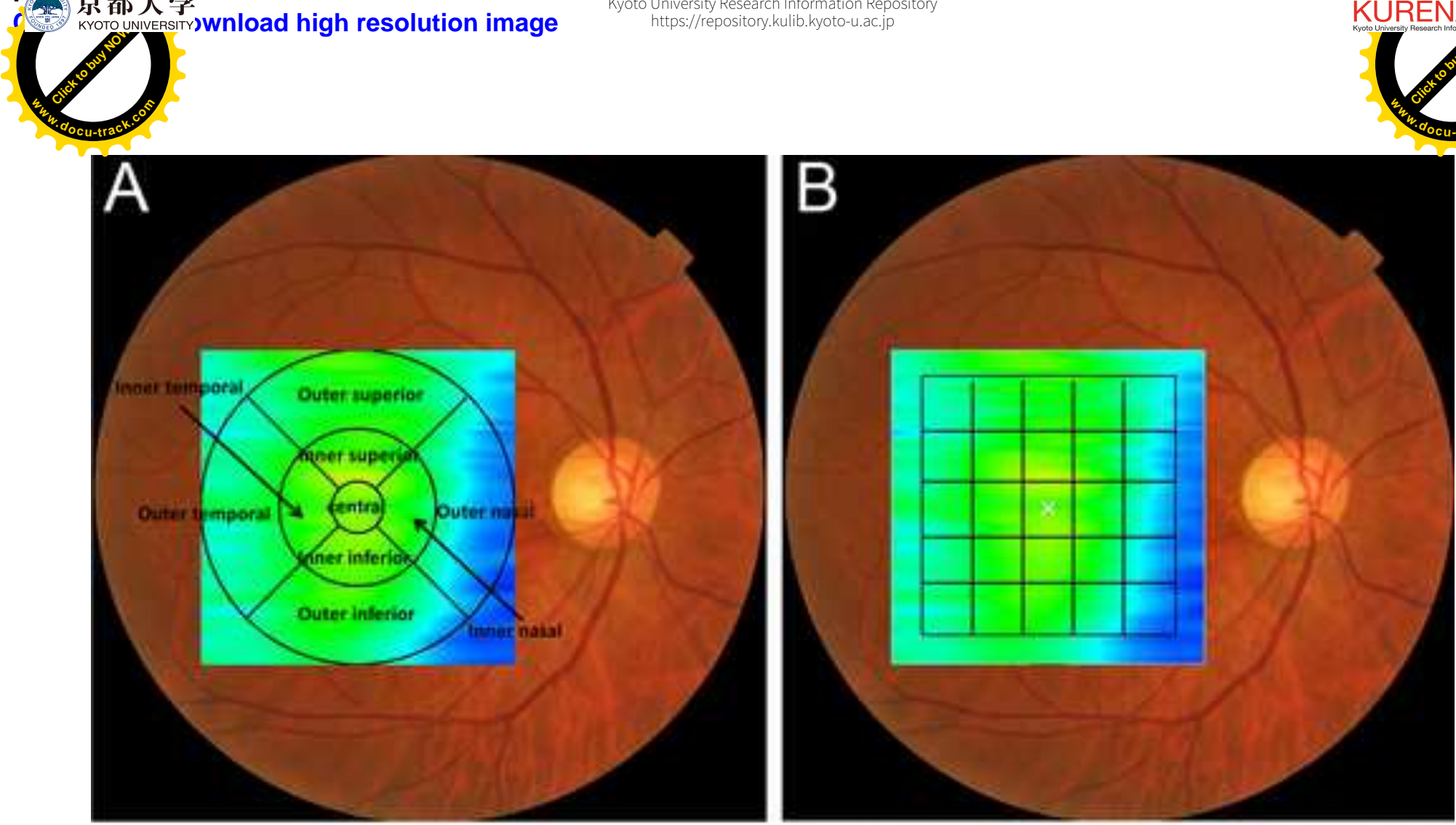

\section{E}

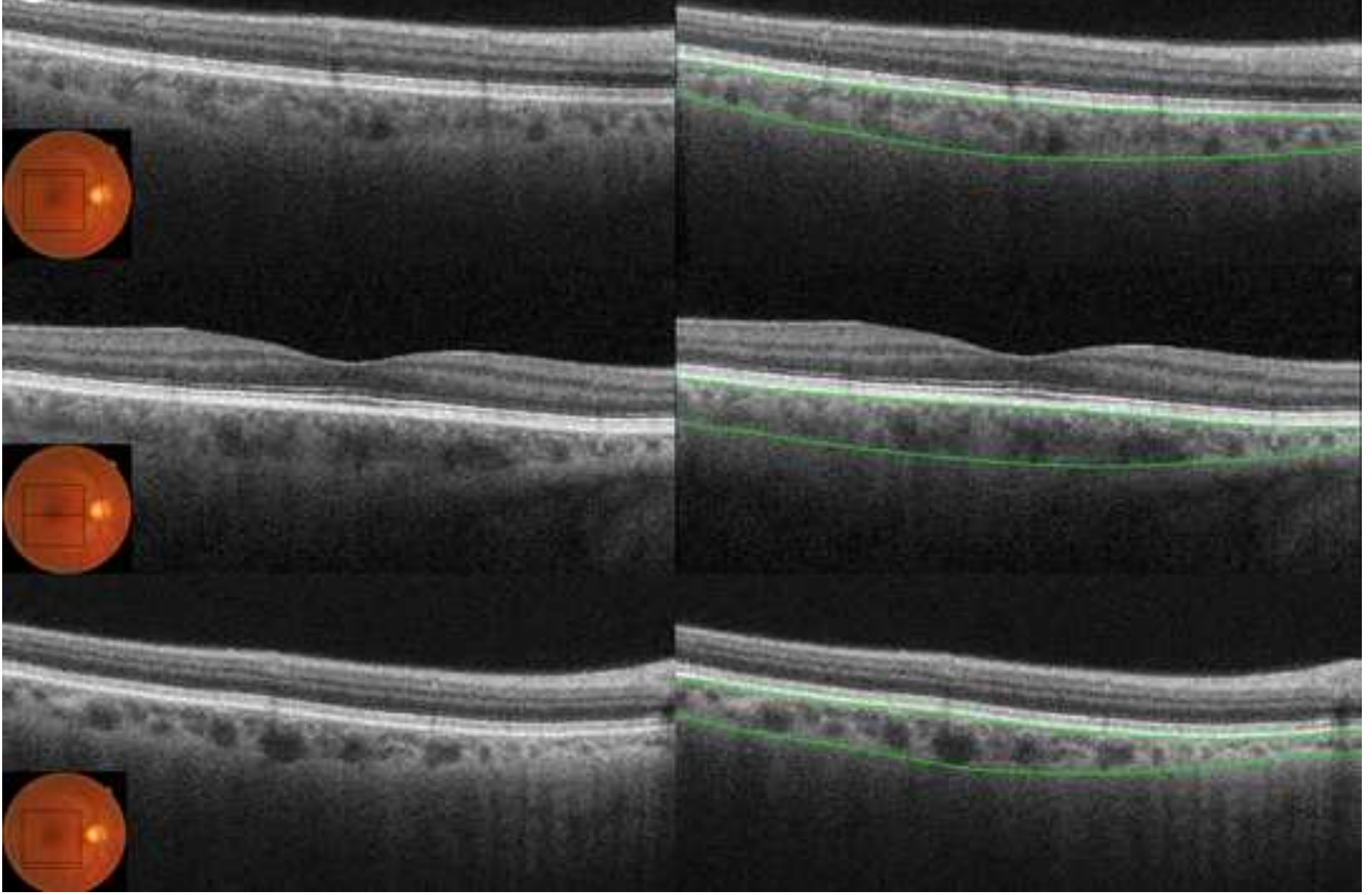


F: 京都大学

A Self-archived copy in

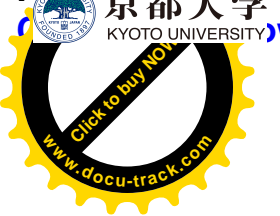

\section{nload high resolution image}
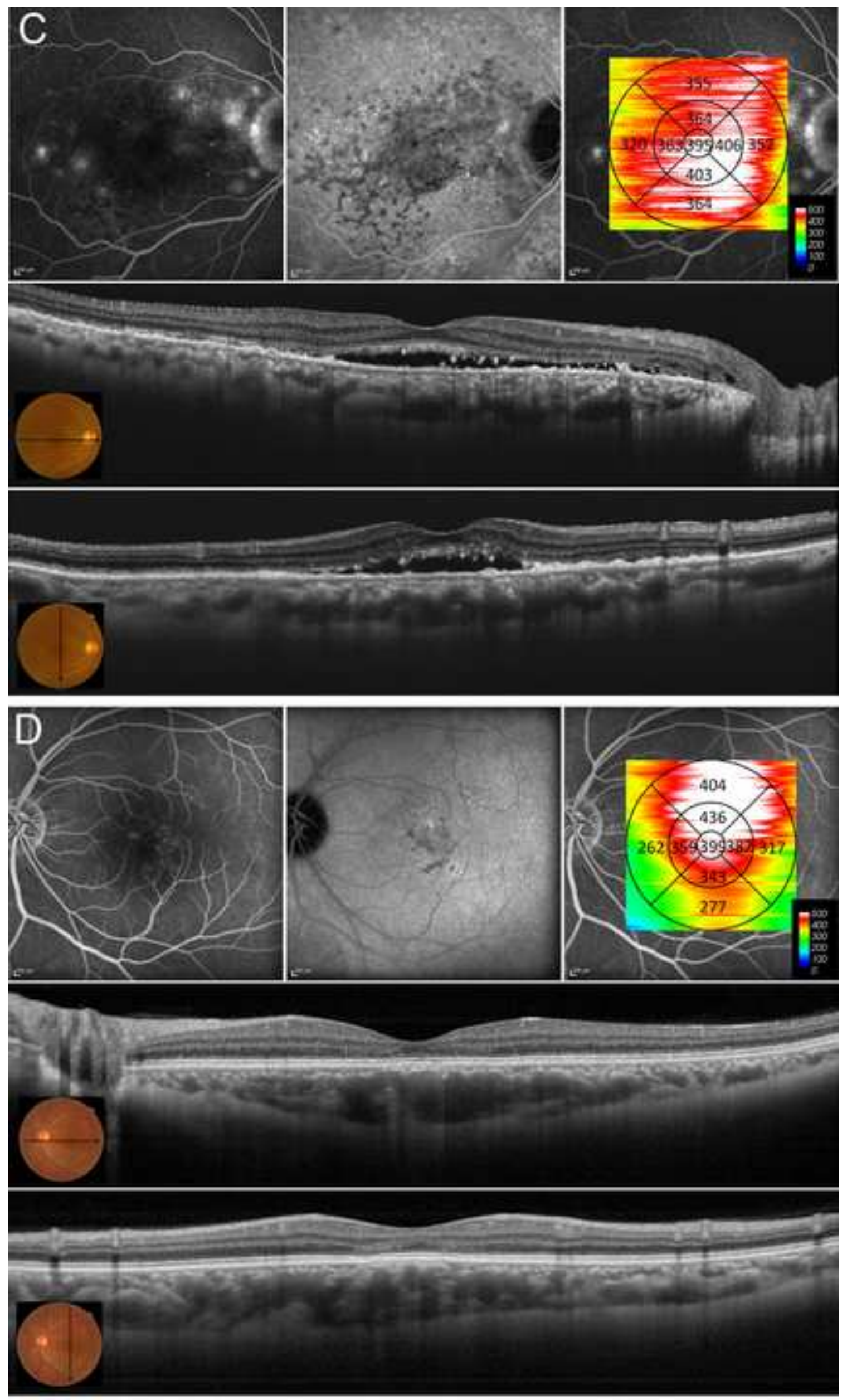

京都大学学術情教りホジトリく

KURENAI 红
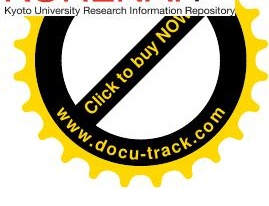


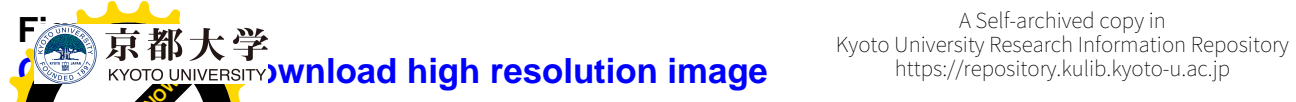

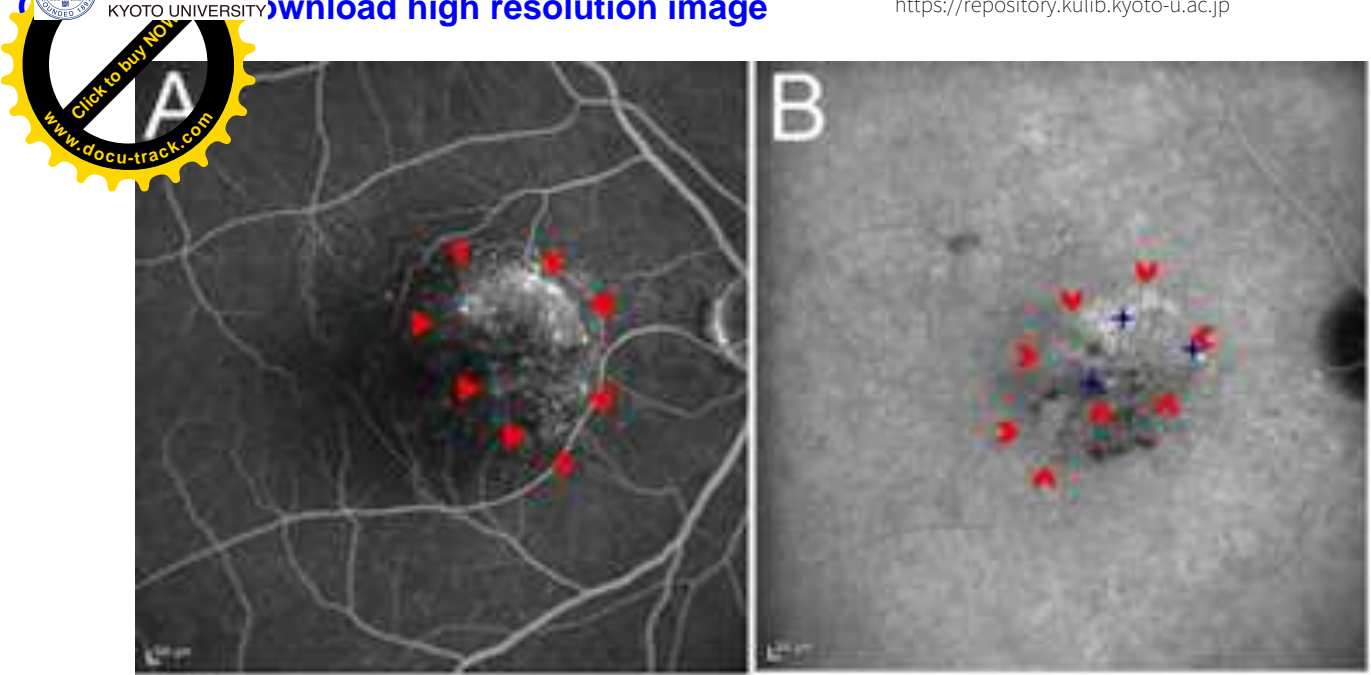

6
KURENAI 红

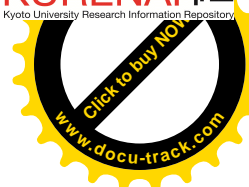

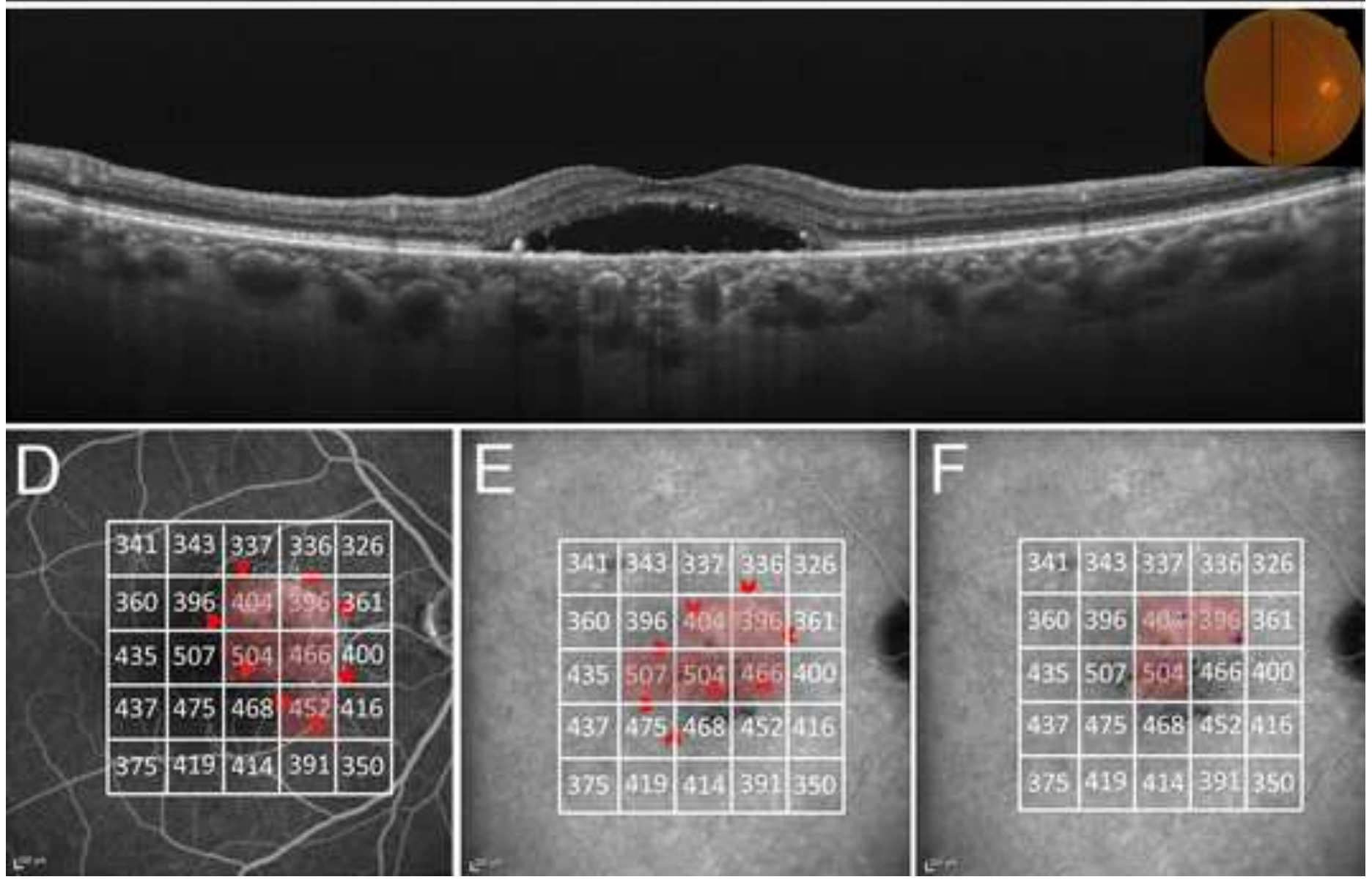

2.
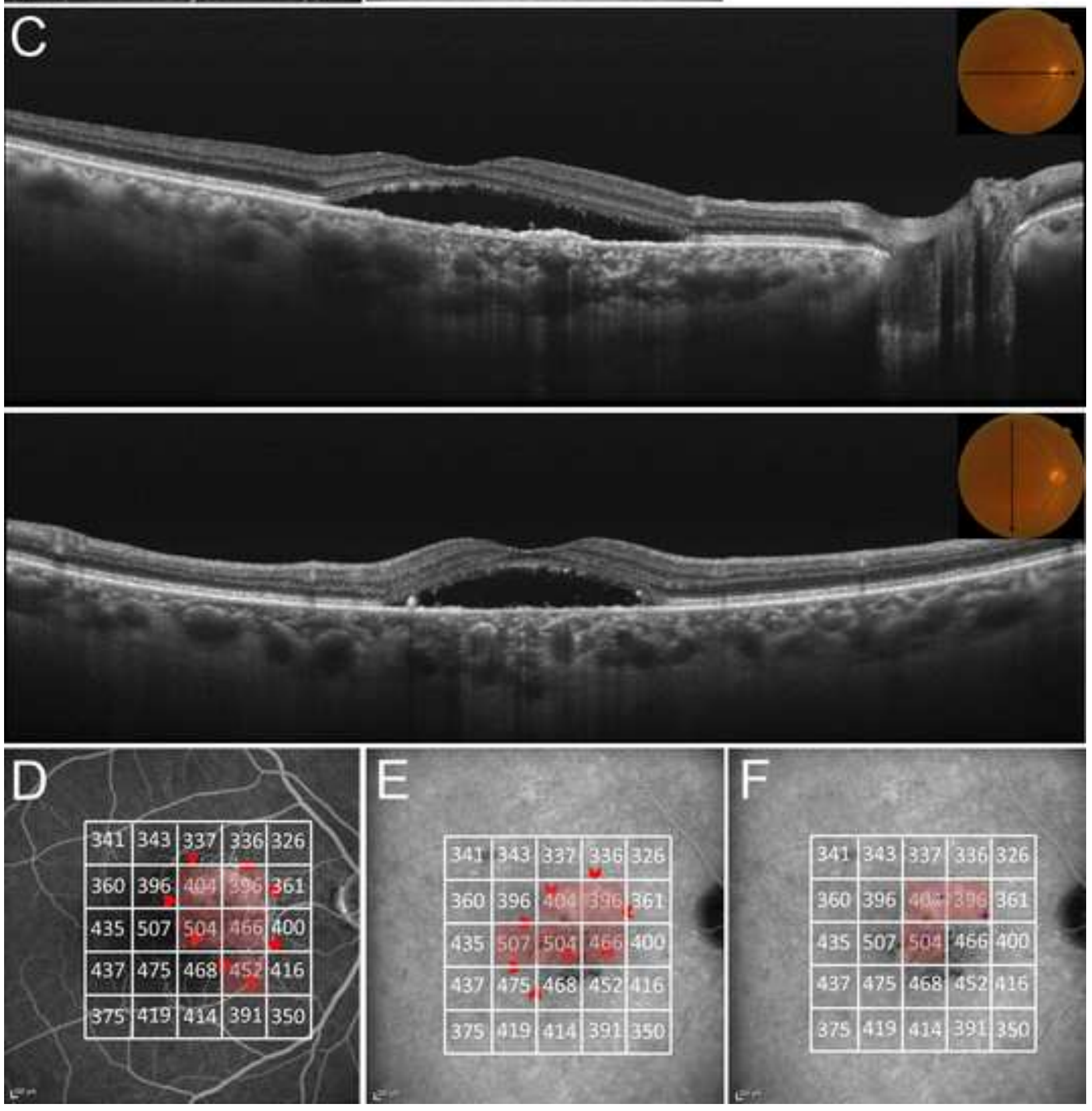

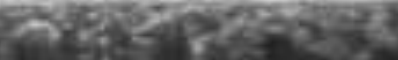

\begin{tabular}{|l|l|l|l|l|}
\hline 350 & 396 & 409 & 586 & 361 \\
\hline 435 & 507 & 504 & 466 & 400 \\
\hline 437 & 475 & 468 & 452 & 416 \\
\hline 375 & 019 & 414 & 391 & 350 \\
\hline
\end{tabular}

\begin{tabular}{|l|l|l|l|l|}
\hline 341 & 343 & 337 & 336 & 326 \\
\hline 360 & 396 & 409 & 398 & 361 \\
\hline 435 & 507 & 504 & 466 & 400 \\
\hline 437 & 475 & 468 & 452 & 416 \\
\hline 375 & 419 & 414 & 391 & 350 \\
\hline
\end{tabular}
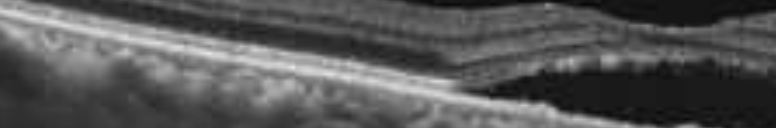

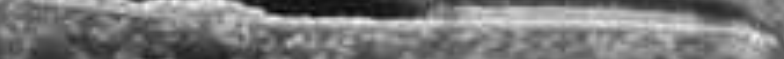


Figure 1. A choroidal thickness map obtained by high-penetrating swept source-optical coherence tomography in a normal eye. (A) Three-dimensional raster scan covered a $6 \times 6 \mathrm{~mm}$ area centered on the fovea. The Early Treatment Diabetic Retinopathy Study sector was applied to the scanned area and the mean choroidal thickness of 9 sectors was measured. (B) The $5 \times 5$ $\mathrm{mm}$ squared grid sector was applied to the scanned area, and the mean choroidal thickness of 25 square areas was measured. (C) B-scan images at difference levels of the raster scan. Green lines indicate the segmentation lines that show Bruch's membrane (outer side of the retinal pigment epithelium and Bruch's membrane complex) and the chorioscleral border. 
Figure 2. Macular choroidal thickness in each subtype of central serous chorioretinopathy (CSC). (A) Active classic CSC. (Top, left) Fluorescein angiography (FA) shows an ink blot pattern of leakage; (Top, middle) Indocyanine green angiography (IA) shows choroidal hyperpermeability and punctate hyperfluorescent spots; (Top, right) Choroidal thickness map shows increased choroidal thickness in the whole macula. ( $2^{\text {nd }}$ row, bottom) SS-OCT images $(12 \mathrm{~mm}$ ) of the fovea show serous retinal detachment and a thick choroid. (B) Active chronic CSC. (Top, left) FA shows broad areas of granular hyperfluorescence during FA associated with many indistinct areas of leakage; (Top, middle) IA shows choroidal hyperpermeability and punctuate hyperfluorescent spots; (Top, right) Choroidal thickness map shows increased choroidal thickness in the whole macula. ( $2^{\text {nd }}$ row, bottom) SS-OCT images $(12 \mathrm{~mm})$ through the fovea show serous retinal detachment involving the fovea, thin central retina, and thick choroid. (C) Active multifocal posterior pigment epitheliopathy. (Top, left) FA shows multiple massive leakages from the RPE; (Top, middle) IA shows several areas of choroidal hyperpermeability and punctuate hyperfluorescent spots; (Top, right) Choroidal thickness map shows increased choroidal thickness in the whole macula. $\left(2^{\text {nd }}\right.$ row, bottom) SS-OCT images $(12 \mathrm{~mm}$ ) through the fovea show serous retinal detachment and a thick choroid. (D) Resolved classic CSC. (Top, left) FA shows a window defect and no remarkable leakage; (Top, middle) IA shows several areas of choroidal hyperpermeability and punctuate hyperfluorescent spots; (Top, right) Choroidal thickness map shows increased choroidal thickness in the whole macula. $\left(2^{\text {nd }}\right.$ row, Bottom) SS-OCT images $(12 \mathrm{~mm})$ through the fovea show no serous retinal detachment, but the presence of a thick choroid. 
Figure 3. Comparison of the mean choroidal thickness and angiographic findings in an eye with active chronic central serous chorioretinopathy. (A) Fluorescein angiography (FA) shows diffuse leakage (red triangle). (B) Late-phase indocyanine angiography (IA) shows choroidal hyperpermeability (red arrowheads) and punctate hyperfluorescent spots (blue stars). (C) SS-OCT images $(12 \mathrm{~mm}$ ) show serous retinal detachment in the fovea and a thick choroid. (D) The mean choroidal thickness in areas with FA leakage indicted by red (442.5 $\mu \mathrm{m})$ was greater, compared with non-FA leakage areas $(397.1 \mu \mathrm{m})$. (F) The mean choroidal thickness in areas with choroidal hyperpermeability indicated by red $(458.7 \mu \mathrm{m})$ was greater, compared with the non-hyperpermeability areas $(387.2 \mu \mathrm{m})$. (G) The mean choroidal thickness in areas with punctuate hyperfluorescent spots indicated by red $(434.7 \mu \mathrm{m})$ was greater, compared with non-punctate hyperfluorescent areas $(400.2 \mu \mathrm{m})$. 
Figure 4. Comparison of the mean choroidal thickness and angiographic findings in an eye with resolved chronic central serous chorioretinopathy. (A) Fluorescein angiography (FA) shows a window defect in an area with retinal pigment epithelium alteration, and no remarkable leakage. (B) Early phase indocyanine angiography (IA) shows filling delay in choroidal arteries and choriocapillaris (red arrows). (C) Late-phase IA shows choroidal hyperpermeability (red arrowheads) and punctate hyperfluorescent spots (blue stars). (D) SS-OCT images $(12 \mathrm{~mm}$ ) show no serous retinal detachment, but show a thick choroid. (E) The mean choroidal thickness in areas with filling delay indicated by red $(247.1 \mu \mathrm{m})$ was almost equal to that in non-filling delayed areas $(243.5 \mu \mathrm{m})$. $(\mathrm{F})$ The mean choroidal thickness in areas with choroidal hyperpermeability indicated by red $(302.7 \mu \mathrm{m})$ was greater than that in non-hyperpermeability areas $(223.4 \mu \mathrm{m}) .(\mathrm{G})$ The mean choroidal thickness in areas with punctuate hyperfluorescent spots indicated by red $(303.5 \mu \mathrm{m})$ was greater than that in non-punctate hyperfluorescent areas $(240.6 \mu \mathrm{m})$. 